\title{
Del aprendizaje a la globalización: la influencia del mercado y las instituciones en las multinacionales chinas
}

\author{
Lilliana Lorena Avendaño Miranda*
}

\begin{abstract}
Resumen
La literatura económica convencional sobre la inversión extranjera directa (IED) ha tipificado la conducta estratégica de las empresas multinacionales a través del prisma de la teoría de la firma. Sin embargo, la IED proveniente de economías en desarrollo ha revelado la existencia de empresas multinacionales cuyo comportamiento difiere de lo pronosticado por las teorías convencionales debido a la intervención de instituciones gubernamentales, como en el caso chino. Tomando en consideración dicho señalamiento, este trabajo intenta desvelar, a través de las teorías convencionales e institucionales, los factores que han influido en la conducta estratégica de las multinacionales chinas desde las reformas económicas de 1978 hasta la actualidad.
\end{abstract}

Palabras clave: empresas multinacionales, conducta de la firma, instituciones, imperfecciones de mercado, China.

Clasificación JEL: B52, D22, D23, F23.

\section{INTRODUCCIÓN}

En la literatura económica existe un cuerpo muy importante de teorías que consideran a la inversión extranjera directa (IED) como el resultado de motivaciones puramente empresariales y de mercado, las cuales constituyen parte esencial del conjunto teórico dedicado a explicar dicha inversión. Tales teorías, emanadas principalmente de las investigaciones de Hymer, Casson y Buckley y Dunning, se centran en la firma, en su conducta estratégica frente a las imperfecciones del mercado y en sus decisiones de localización. ${ }^{1}$ Esas teorías han sido amplia-

Manuscrito recibido en agosto de 2012; aceptado en mayo de 2013.

* Profesora de la Facultad de Ciencias Administrativas y Sociales de la Universidad Veracruzana, México, $<$ lorena_aven@hotmail.com>. La autora agradece los comentarios y sugerencias de la Dra. Clara García Fernández-Muro, así como de los dictaminadores anónimos de la revista.

1 Una extensa exposición sobre las teorías de la firma puede encontrarse en Kantarelis (2007). 
mente utilizadas para explicar los motivos de la IED, por lo que en este trabajo se denominarán teorías convencionales.

No obstante, la entrada en el escenario internacional de las empresas japonesas en un primer momento, de las firmas provenientes del sudeste asiático más tarde y más recientemente de las empresas chinas, ha desvelado la necesidad de ampliar la teoría económica para dar cabida a otras posibles razones que expliquen la expansión mundial de la IED proveniente de países en desarrollo, tarea que han acometido varios autores a partir del prisma institucional como explicación alternativa a los enfoques mencionados.

Esta necesidad surge del reconocimiento de las características diferenciadas de las firmas de países en desarrollo que en la mayoría de los casos no han observado una conducta estratégica semejante a la de las provenientes de países desarrollados, principalmente por razones institucionales. En el caso chino este hecho es en particular cierto debido al papel activo del gobierno en el establecimiento de leyes y organismos de promoción para impulsar la emisión de IED, lo que ha dado lugar al surgimiento de multinacionales no convencionales. Por esta razón, diversos investigadores consideran pertinente que en el caso mencionado, además de los motivos convencionales, las instituciones también sean consideradas un determinante de la IED del país asiático.

Tomando en cuenta dichos señalamientos, en esta investigación se pretende analizar, a través de la óptica de la teoría de la firma y de las teorías institucionales, en primer lugar, de qué forma han influido en las multinacionales chinas las imperfecciones provocadas por el mercado y, en segundo lugar, confirmar si a lo largo de su evolución la conducta estratégica de esas firmas ha sido influida por las instituciones y, si es así, de qué forma ha sucedido. Para alcanzar dichos objetivos, el horizonte temporal de este análisis abarcará desde las reformas económicas de 1978 hasta el presente, realizándose mediante algunos elementos de las teorías consideradas como convencionales (relacionados principalmente con los fallos de mercado). Conjuntamente, el análisis institucional ${ }^{2}$ se realizará a

\footnotetext{
2 Las teorías convencionales se eligieron debido a que estudian la IED como una iniciativa empresarial ligada a decisiones empresariales y, de alguna forma, libre de otras influencias que no sean las del mercado, lo que representa un contrapunto con respecto a las teorías institucionales que presuponen la intervención de las instituciones en el comportamiento de los agentes económicos. Es decir, a partir de las teorías convencionales se podrá obtener una visión no intervencionista de la conducta de las firmas frente a la visión intervencionista de las teorías institucionales.
} 
partir de las instituciones exógenas de carácter formal ${ }^{3}$ emanadas del gobierno que intervenga de alguna forma en la emisión de IED.

Al hablar de instituciones públicas algunos autores se refieren al gobierno y otros al Estado. En esta investigación se respetarán dichos términos cuando se haga mención directa a sus trabajos, pero en el resto del análisis se utilizará el término gobierno cuando se mencionen las instituciones emanadas del Estado o cuando se aluda al ejercicio del poder y la conducción política del Estado.

Este trabajo está organizado de la siguiente forma: primero, se presentará una breve revisión teórica referente a las teorías de la firma e institucionales y su relación con la IED. Segundo, se expondrá un ejemplo representativo de una empresa china y su proceso de internacionalización, para entender mejor el contexto institucional y de mercado que rodeó el advenimiento de buena parte de tales empresas. Tercero, se examinará la conducta estratégica de las multinacionales chinas ante las imperfecciones de mercado. Cuarto, se estudiará cómo afectan las instituciones al mercado interno y a las empresas. Quinto, se señalará cuál ha sido la respuesta de las firmas ante la influencia institucional. Por último, se presentarán las conclusiones.

\section{BREVE REVISIÓN TEÓRICA}

Hasta mediados de la década de los setenta la IED fue una actividad acometida de forma casi exclusiva por empresas multinacionales (EMN) de países desarrollados. El desempeño de éstas sirvió como base para establecer algunas de las teorías económicas generalmente aceptadas para explicar la IED. Las primeras aportaciones en este campo surgieron de la pluma de Hymer (1976), quien diferenció dicha inversión de la de cartera, estableciendo que la primera se explica por razones de control y de explotación de ventajas particulares de la firma, mientras que la segunda existe debido a la diferencia de los tipos de interés en los diversos países.

Otras teorías generalmente aceptadas están representadas por los trabajos de Buckley y Casson (1976) y Dunning (1981; 1988; 1993), que grosso modo podrían

\footnotetext{
${ }^{3}$ Como instituciones exógenas se entenderán aquellas generadas fuera de la firma, en contraposición a las instituciones internas derivadas de la estructura organizacional que surgen cuando una empresa decide coordinar sus recursos por medio de mercados internos, tal como lo enuncian Coase (1937) y Willamson (1989). Como instituciones formales se entenderán las leyes, reglamentos, constituciones, contratos y todas aquellas restricciones escritas según lo enunciado por North (1995).
} 
resumirse en que la IED ocurre cuando una firma busca maximizar sus beneficios explotando por sí misma sus ventajas de propiedad en mercados extranjeros con estructura oligopólica y suficiente atractivo para el desarrollo de su actividad. El argumento central de las teorías generalmente aceptadas o teorías convencionales (como se les denomina en esta investigación) es que la internacionalización de una firma descansa fundamentalmente en su capacidad de beneficiarse de sus ventajas de propiedad en mercados imperfectos. A su vez, las imperfecciones de mercado que propician o determinan la IED, de acuerdo con dichas teorías, son: oligopolios, costos de transacción, asimetrías de conocimiento, economías de escala y bienes diferenciados.

Cabe subrayar que Dunning $(1981 ; 1988 ; 1993)$ desarrolló un modelo que trata de integrar elementos de las teorías de la organización industrial, de la firma y de la localización. Así, el paradigma ecléctico de Dunning establece que el modelo de producción internacional está determinado por tres tipos de ventajas: de propiedad $(\mathrm{O})$, de localización (L) y de internalización (I). También afirma que la IED puede estar motivada por la búsqueda de mercados, de recursos, de eficiencia o de activos estratégicos. Dado que las teorías convencionales basan sus principios en el estudio de la conducta de multinacionales de países desarrollados (o EMN convencionales), dicha conducta se convirtió en el modelo de estudio para establecer las pautas de internacionalización de una firma y determinar su conducta estratégica.

Con respecto a las teorías institucionales (no convencionales), las investigaciones de North $(1979 ; 1993 ; 1995)$ proporcionan elementos muy valiosos para analizar la relación entre el gobierno (y sus organismos), la legislación y los aspectos económicos de un país (en este caso la IED), estableciendo un nexo con las teorías convencionales.

North (1993) explica que, a diferencia del pasado, cuando se pretendía remplazar a la teoría neoclásica, en el presente la nueva economía institucional (NEI) intenta incorporar la teoría de las instituciones a la teoría económica para ampliarla. El enfoque de la NEI propuesto encaja en la teoría neoclásica porque la concibe como una teoría en la que las elecciones están sujetas a limitaciones; la teoría del precio es una parte primordial en el análisis institucional y los cambios en los precios relativos son un factor muy significativo que induce el cambio en las instituciones. Además, la NEI no sólo toma en cuenta el concepto de costo de producción neoclásico sino que incorpora los costos de transacción de la teoría coasiana y los hace parte central de su análisis. (North, 1993; Rutherford, 2001). 
Entre las diversas instituciones existentes los derechos de propiedad destacan ya que están estrechamente relacionados con los costos de transacción. La emergencia de nuevos derechos de propiedad evidencia cambios en los valores económicos ligados al desarrollo de nuevas tecnologías y a la apertura de nuevos mercados para los cuales los antiguos derechos de propiedad no están suficientemente preparados (Demsetz, 1967). Estos últimos suelen expresarse por medio de leyes y contratos que los agentes económicos deben cumplir; al mismo tiempo, es necesaria la creación de organismos que vigilen dicho cumplimiento.

North (1993) hace una diferenciación entre instituciones y organismos, definiendo a las primeras como la estructura de incentivos de los individuos que interactúan en sociedad, mientras que las segundas son grupos unidos por un mismo fin: el de maximizar sus beneficios. De esta forma, los organismos y las instituciones políticas y económicas son elementos determinantes fundamentales del desempeño económico a largo plazo. Dado que las organizaciones buscan la maximización de sus beneficios, tenderán a modificar la estructura institucional existente si ello les permite alcanzar sus objetivos (North, 1995). Dichos cambios pueden dar lugar a un aumento o a una disminución en los costos de transacción.

Las instituciones proporcionan la clave para comprender la relación entre la política y la economía, y a su vez, entender las consecuencias de esta relación para el crecimiento económico. El Estado es el máximo órgano de establecimiento y vigilancia del marco institucional en que se realizarán los intercambios, lo cual es muy conveniente porque lo hace a costos menores que si lo hiciera un particular, aunque también es posible que cree instituciones ineficientes que den lugar a un efecto contraproducente, aumentando los costos de transacción (North, 1995).

La maximización de beneficios tiene que ajustarse a las "reglas del juego" impuestas por el Estado; éstas a su vez están ideadas para facilitar las actividades económicas y disminuir los costos de transacción, los cuales inciden en los costos totales y éstos en los beneficios. Al disminuir los de transacción, los totales disminuyen y los beneficios aumentan, lo mismo que los impuestos que deben pagar los actores económicos, lo que redunda en un beneficio para el Estado. Sin embargo, puede suceder que, con la finalidad de aumentar sus propias rentas o beneficiar a determinados grupos, el Estado establezca derechos de propiedad que en lugar de alentar, obstaculicen el crecimiento económico (North y Thomas, 1973; North, 1979; North, 1995). 
El interés por promover el crecimiento económico ha llevado a los gobiernos de los estados a establecer políticas para el aumento de la competitividad de las empresas nacionales, para la promoción de exportaciones y para el fomento de la IED. Asimismo han creado y modificado leyes, normas y organismos gubernamentales con la finalidad de contar con un marco institucional que les permita lograr sus objetivos económicos, políticos y sociales. El fin ulterior de esta estrategia es prolongar su permanencia en el poder. De esta manera, North (1995) establece una relación entre el gobierno, la creación de instituciones y organismos para el alcance de ciertos objetivos y la permanencia en el poder.

Scott (1985) reconoce la importancia del Estado en la creación de un clima propicio para los negocios. Un ejemplo de ello son las firmas de países del este de Asia, que experimentaron un extraordinario crecimiento en escala internacional gracias a la decidida participación de sus gobiernos en el diseño de una estrategia nacional para promover las inversiones, la investigación y desarrollo (I+D), los préstamos baratos, la protección de los mercados en una etapa inicial y una estrecha colaboración con las empresas para lograr la calidad y productividad del primer mundo. De esta forma el autor establece una relación entre el Estado, las instituciones y las empresas, en donde el primero crea una serie de instituciones para favorecer la actividad económica, permitiendo que las empresas nacionales crezcan más allá de sus fronteras.

Por su parte, Giddy y Young (1982: 62) denominan 'EMN no convencionales' a las que no provienen de países desarrollados ni cuentan con las características típicas de esas empresas, sino que son pequeñas productoras de manufacturas no diferenciadas, de bajo nivel tecnológico que realizan joint ventures (pero tienden a retener el control) para internacionalizarse y que en la mayoría de los casos están constituidas por inversiones que fluyen hacia otros países en desarrollo, por lo general cercanos geográficamente.

Muchas de las EMN no convencionales, a su vez, destacan por ser empresas estatales (en adelante EE), por haber sido fundadas o bien adquiridas por el gobierno. En cualquier caso, dichas empresas abarcan un amplio rango de industrias y han representado un papel muy importante en los países en desarrollo, tal como lo subrayan Jones (1975) y Sheahan (1976).

Las EE suelen ser utilizadas por el gobierno como un instrumento de política industrial para desarrollar sectores rezagados o presionados por la competencia externa, así como para crear y mantener empleos. Conviene señalar que aunque las EE suelen administrarse de forma independiente, lo cierto es que deben res- 
ponder a las instancias estatales. De hecho, con frecuencia sus objetivos están lejos de relacionarse con la generación de beneficios y más bien tienen que ver con asuntos de interés político (Walters y Monsen, 1977).

Además de Scott (1985) y Giddy y Young (1982), otros autores como Lecraw (1983), Heenan y Keegan (1979) y Lall (1983) advirtieron la emergencia de la IED proveniente de países en desarrollo, caracterizando un nuevo tipo de EMN que mostraba rasgos distintos a los de sus contrapartes convencionales estudiadas hasta ese momento. A pesar de que las nuevas EMN poseían características que claramente las diferenciaban de las convencionales, los diversos intentos para explicar su comportamiento se enmarcaron dentro de las teorías económicas generalmente aceptadas considerando que su desempeño podía explicarse a partir de las teorías de IED existentes hasta el momento.

Es decir, no se profundizó en los aspectos que habrían dado lugar a la emergencia de multinacionales de países en desarrollo, sino que sólo se adaptaron algunas de las teorías existentes para poder analizar esas nuevas empresas, por lo que tales explicaciones sólo esclarecieron parcialmente el fenómeno.

En el caso particular chino, las investigaciones de Child y Rodrigues (2005) revelaron que la IED de ese origen no seguía algunos de los patrones comunes de la proveniente de países en desarrollo y al mismo tiempo percibieron un vacío que las teorías convencionales no cubrían y propusieron la incorporación de elementos institucionales al análisis convencional para subsanar esa deficiencia. En otras palabras, las instituciones también deberían ser consideradas como un determinante de la IED. Esta propuesta tuvo eco en las investigaciones de Peng (2005), Buckley et al. (2007) y Deng (2009), entre otros.

\section{Beijing Shougang Group:}

\section{LA TRANSICIÓN DE UNA FIRMA NO CONVENCIONAL}

La Shougang Corporation, (más tarde Beijing Shougang Group Co. Ltd.) se fundó en 1919 en Beijing para elaborar productos de acero. Una vez que el Partido Comunista Chino tomó el control de la parte continental del país (1949) la empresa fue dirigida por un comandante del ejército chino, quien rendía cuentas al gobierno de dicha ciudad (Nolan y Yeung, 2001a; 2001b). Al igual que otras EE, la Shougang debía cumplir con una producción y unos beneficios obligatorios marcados por los planes estatales. La mayor parte de la producción la vendía a los precios fijados por el gobierno, pero $15 \%$ de la misma la podía 
ofrecer por encima de éstos en el marco del dual-track system ${ }^{4}$ implementado en la década de los ochenta.

Con el comienzo de las reformas económicas, en 1978 gran parte de la industria se orientó hacia las manufacturas baratas, con poco contenido tecnológico y escasa calidad; la Shougang Corporation no fue la excepción. Entre 1978 y 1994 el gobierno de la ciudad de Beijing ejerció como Consejo de Administración de la firma. El gobierno central controlaba tanto los salarios como los despidos y los empleados en puestos directivos no recibían compensaciones especiales ni dividendos. Además, se obligaba a la empresa a crecer antes que a recurrir al despido para eliminar el exceso de trabajadores. De ello derivó una estrategia de diversificación como forma de disminuir el exceso de personal en las plantas que permeó en la mayoría de las industrias de esa época (Nolan y Yeung, 2001b).

Al igual que sucedió con otras EE (Nolan y Zhang, 2002) en 1983 la Shougang se fusionó con 17 acereras, pero a pesar de ello sus activos siguieron siendo tan obsoletos que se le conocía como el "museo de historia de la metalurgia" (Nolan y Yeung, 2001a: 446). Ante tal situación, la empresa obtuvo el permiso para invertir en maquinaria y equipo importado con el fin de renovar sus plantas a condición de que continuara generando los beneficios fijados por el plan estatal. Durante la década de los ochenta la empresa poseía una línea de productos limitada, unas afiliadas que producían escasos beneficios (e incluso pérdidas), exceso de trabajadores y se encontraba bajo el control presupuestario del gobierno. Cuando la firma intentó expandir operaciones en otras provincias del país el gobierno central no dio su consentimiento.

A partir de 1990 los controles presupuestarios se relajaron y la firma obtuvo ayuda financiera del gobierno e incluso pudo establecer su propio banco: el Huaxia Bank. En esa época emprendió una serie de fusiones y adquisiciones (FyA) nacionales coordinadas desde el gobierno central de modo que a principios de los años noventa poseía 157 plantas y 65 joint ventures, convirtiéndose en la cuarta empresa en escala nacional por el total de sus ventas. Para 1993 ya contaba con siete H-share companies ${ }^{5}$ en Hong Kong y en 1995 estableció una

\footnotetext{
4 Sistema que permitía a las empresas vender sus productos utilizando dos tipos de precios: uno fijado por el gobierno (generalmente más bajo) y otro por el mercado (generalmente más elevado). Con ello se pretendía sustituir de modo paulatino la economía planificada por una de mercado (Naughton, 2007).

5 Empresas establecidas en la China continental y autorizadas por la China Securities Regulatory Commission (CSRC) para cotizar en la bolsa de Hong Kong.
} 
empresa en Singapur para controlar sus exportaciones en Asia. En 1994 ya tenía inversiones y alianzas en Asia, América, Europa y Oriente Medio, operaciones que expandió a 13 países. Sin apenas experiencia, la empresa tomó varias decisiones equivocadas y se endeudó en exceso, lo que finalmente la llevó a la quiebra, y por ello tuvo que ser rescatada por el gobierno chino a finales de los años noventa (Nolan y Yeung, 2001b; Movshuk, 2004).

A partir de 1995 la empresa comenzó a experimentar transformaciones importantes: introdujo un moderno sistema de gerencia, estableció un consejo de administración, gerentes y un comité de supervisión, se dio personalidad jurídica a las empresas del grupo y se fijaron precios de mercado para las transacciones intragrupales (Nolan y Yeung, 2001b). A mediados de los años noventa el gobierno de Beijing resolvió cambiar su perfil para basarlo en el conocimiento y en los productos de alto contenido tecnológico. A pesar de sus problemas financieros, en 1996 la firma se convirtió en una de las EMN no convencionales líder en el mercado internacional (Nolan y Yeung, 2001b). Conforme al acuerdo de 1997 del Partido Comunista, se determinó que la Shougang, junto con otras tres empresas del acero (Baogang, Angang y Wugang) se convirtieran en campeones nacionales para competir con sus homólogas de clase mundial (Nolan y Yeung, 2001a). En 1997 la firma poseía 71 empresas afiliadas, de las cuales 25 presentaban pérdidas que a su vez se compensaban con las ganancias producidas por las filiales extranjeras (Nolan y Yeung, 2001b).

Una vez comenzado el nuevo siglo la restructuración del grupo continuó con la finalidad de eliminar para el 2005 todas las empresas con pérdidas, establecer nuevos centros de $\mathrm{I}+\mathrm{D}$, elevar la calidad de sus productos, así como aumentar sus ventas totales y su margen de beneficios (Nolan y Yeung, 2001b). En la actualidad cuenta con 148 empresas e institutos de investigación dentro y fuera de China, además de que posee negocios en los sectores minero, manufacturero, de producción de gas y energía eléctrica, inmobiliario, de la construcción y transporte marítimo, por mencionar algunos.

\section{LA CONDUCTA ESTRATÉGICA DE LAS MULTINACIONALES CHINAS ANTE LAS IMPERFECCIONES DE MERCADO}

La historia del Beijing Shougang Group es la misma que la de una buena parte de las Ee chinas que a partir de 1978 protagonizaron algunos de los cambios más importantes experimentados por el país. Conocer el proceso evolutivo de dichas EE permite comprender la historia de su internacionalización; de ahí 
que para fines de análisis en esta investigación se distinguirán tres fases principales de evolución: a) la de aprendizaje e internacionalización temprana; $b$ ) la de crecimiento en el mercado internacional, y c) la de expansión global. ${ }^{6}$ Con el análisis de cada una de las fases se espera esclarecer el comportamiento que han mostrado las firmas ante las imperfecciones de mercado mencionadas en la revisión teórica. Asimismo se espera conocer si las instituciones también han afectado dicho comportamiento y de qué modo.

\section{Primera fase: aprendizaje e internacionalización temprana}

Las primeras inversiones chinas en ultramar se orientaron al comercio (para asegurar la obtención de recursos naturales y de tecnología, principalmente) por lo que fueron las empresas comercializadoras y algunos institutos de investigación los que comenzaron a localizarse en el extranjero al amparo de las Fifteen Measures of Economic Reform de 1978 que autorizaron dichas inversiones. Una vez aprobada en 1984 la Circular Concerning Approval Authorities and Administrative Principles for Opening up Non-Trade Joint Venture Overseas que permitía a empresas no comerciales invertir en el extranjero, otras EE comenzaron a invertir fuera del país (Buckley et al., 2007). Durante los primeros años de la apertura las empresas estatales eran grandes aparatos burocráticos sometidos a diversas limitaciones y a la competencia de las township and village enterprises (TVE) y de las empresas privadas que gozaban de una relativa libertad que las EE no tenían (Naughton, 2007). Como se verá a continuación, la fragmentación del mercado y la dificultad para generar ventajas $\mathrm{O}$ (sobre todo en lo referente la protección de la propiedad intelectual) y ventajas I marcaron a las firmas en esta fase.

\section{Fragmentación del mercado}

Esta situación surgió debido a tres razones principalmente: en primer lugar, como parte de su política de descentralización, ${ }^{7}$ el gobierno central responsabilizó a provincias y municipios de su desarrollo económico.

6 Estas fases no están circunscritas a un periodo determinado y su duración varía en cada empresa. Sin embargo, puede decirse que, en general, la primera fase abarca la década de los años ochenta y principios de la siguiente; la segunda va de mediados de los años noventa a los primeros de la década de 2000, y la tercera de entonces hasta la actualidad.

7 La política de descentralización formó parte primordial de las reformas emprendidas en la década de los ochenta, el monopolio industrial ejercido por el gobierno se relajó y se permitió la entrada de nue- 
En segundo lugar, entre 1980 y principios de 1990 algunas EE comenzaron a ser administradas por los gobiernos provinciales y locales como parte de dicha política de descentralización. Al mismo tiempo, el desempeño de las EE comenzó a medirse en función de los beneficios generados y ya no de la producción fijada de acuerdo con los planes estatales. Ello tuvo un efecto muy importante en las provincias y municipios ya que debían hacerse cargo de las prestaciones laborales (educación, salud y pensiones), por lo que estaban especialmente interesadas en que las EE generaran beneficios (Naughton, 2007).

En tercer lugar, las provincias podían negociar una tasa fija de impuestos, de modo que podían retener todos los ingresos que excedieran esa cantidad (Boisot y Meyer, 2008). Por tanto, la obligación de velar por su propio desarrollo, la responsabilidad de pagar las prestaciones de los trabajadores y la posibilidad de retener los ingresos por impuestos recaudados que excedieran la tasa fijada por el gobierno convirtió a las provincias y municipios en sitios altamente protegidos por su miedo a que disminuyeran sus ingresos y su poder.

Esta circunstancia no sólo ha elevado los costos de transacción, obstaculizando el crecimiento horizontal y/o vertical de las firmas, sino que ha elevado también los costos de operación dentro del territorio nacional, lo que dificulta el establecimiento de un servicio de transporte multimodal eficiente, que facilite la cadena de suministro y las operaciones intrafirma.

\section{Dificultad para generar ventajas $\mathrm{O}$}

Ya que su línea de productos y los precios de venta estaban fijados por el gobierno, las EE no podían generar fácilmente ventajas de propiedad. Al mismo tiempo, las instituciones para velar la propiedad privada eran incipientes. En 1982 se emitió la Ley de Marcas y en 1984 la Ley de Patentes con el propósito de establecer las bases para la protección de la propiedad intelectual en China (Trade Lawyers Advisory Group, 2007). Sin embargo este proyecto estuvo lleno de debilidades (Landry, 2008).

vos competidores, muchos de los cuales estaban controlados por los gobiernos locales. Además, las actividades menos importantes para el gobierno fueron descentralizadas, lo mismo que ciertas competencias administrativas y se permitió que las empresas crecieran fuera del plan central. No obstante, aunque en la década de los noventa se mantuvo la división de responsabilidades entre el gobierno central y los gobiernos locales, el control de los recursos se centralizó nuevamente porque el gobierno necesitaba fortalecer sus funciones regulatorias a nivel macro (Naughton, 2007). 
Por ejemplo, la Ley de Marcas no protegía a aquellas que se considerara que discriminaran cualquier nacionalidad, que fueran contrarias a la moral o a las costumbres socialistas o que tuvieran otras influencias "nocivas". La Ley de Patentes no garantizaba la protección de alimentos, bebidas y aromas ni la de los productos farmacéuticos y las sustancias obtenidas por medio de un proceso químico. Esta ley, además, era contraria a los intereses de los nacionales, ya que los ciudadanos sólo podían patentar aquello que hubiesen inventado por su cuenta o mientras trabajaban en una entidad no estatal, lo que era bastante improbable en ese momento (Trade Lawyers Advisory Group, 2007).

El sistema de justicia también presentaba carencias: no habían jueces cualificados, tampoco agencias de registro de patentes y marcas, ni abogados preparados para llevar litigios en esa materia. Ello dejaba en un estado de indefensión a los inventores nacionales frente a las grandes empresas extranjeras con mayor experiencia y recursos (Landry, 2008). Por tanto, uno de los factores que contribuyó a desalentar la inversión en I+D fue la precariedad del marco legal para la protección de derechos. En consecuencia, la mayoría de las firmas chinas se dedicó a imitar y a copiar tecnologías y productos, en vez de desarrollarlos por sí mismas (Deng, 2009).

\section{Dificultad para generar ventajas I}

Las ventajas de internalización no se pudieron desarrollar debido a tres razones primordiales: la fragmentación del mercado, el crecimiento desmesurado de la plantilla de trabajadores y la diversificación ineficiente.

Como se expuso, la competencia entre provincias y municipios para alcanzar mayores beneficios económicos dio lugar a la fragmentación del mercado interno lo que generó altos costos de transacción (Boisot y Meyer, 2008). La fragmentación, a su vez, impedía el crecimiento eficiente de las empresas por medio de la integración horizontal o vertical de actividades. En consecuencia, tampoco tenían acceso a economías de escala.

El crecimiento desmesurado se debió a que se les impusieron una serie de negocios periféricos con el fin de salvaguardar empresas y empleos (Nolan y Zhang, 2002; Naughton, 2007). Tanto el gobierno central como los gobiernos provinciales intervenían en el caso de FyA nacionales (Child y Rodrigues, 2005). De esta forma, su desarrollo no se realizó por la vía de la eficiencia que da la internalización, sino por la de la diversificación y la falta de competitividad. 
Además, la gran cantidad de trabajadores que poseían las EE originó excesivos niveles jerárquicos con los consiguientes problemas de organización y burocratismo (Nolan y Zhang, 2002).

Para resumir, durante la fase de aprendizaje las condiciones de fragmentación del mercado interno y la dificultad para generar ventajas $\mathrm{O}$ e I situaron a las empresas chinas en una posición muy débil, con líneas de productos limitadas, bajo valor añadido y poca calidad (Nolan y Zhang, 2002; Naughton, 2007).

Ante esta situación las empresas estatales tuvieron tres opciones: establecer joint ventures con empresas extranjeras en el mercado nacional o adquirir licencias; establecer joint ventures en el mercado internacional o, en casos excepcionales, realizar inversiones diversificadas. Una cuarta opción fue la estrategia empleada por las firmas de productos de alta tecnología cuyo desarrollo difiere un poco del resto de EE, como se explicará posteriormente.

En el primer caso, algunas empresas lograron establecer en el mercado nacional joint ventures con empresas extranjeras o adquirieron una licencia de producción y servicio de mantenimiento para hacerse con tecnología más moderna así como para asimilar nuevos procesos, formas de organización y gerencia. Tal fue el caso de, por ejemplo, la joint venture entre Nanjing Automobile Group y el grupo Fiat, Shanghai Automotive con Volkswagen y General Motors, o Haier, que adquirió licencias de Leibherr Group y Merloni (Rui y Yip, 2008; Teagarden y Cai, 2008; Deng, 2009).

En el segundo caso, algunas firmas optaron por invertir en el extranjero mediante joint ventures, ya sea para el abastecimiento de petróleo y materias primas o para establecer canales de distribución; no obstante, la falta de experiencia hizo que algunas de esas operaciones fracasaran rotundamente (Wu y Chen, 2001).

Un tercer caso (que en su momento fue excepcional) es el de Sinochem, que a raíz de su exitosa gestión comercial comenzó a ostentar el monopolio en la importación de petróleo, fertilizantes y materias primas. Pero a medida que otras empresas empezaron a establecer sus propias comercializadoras, Sinochem vio mermar su principal negocio, por lo que recibió permiso para realizar inversiones diversificadas en ultramar y transformarse no sólo en una gran multinacional, sino además en la base sobre la que el gobierno chino experimentó para más adelante autorizar inversiones semejantes a otras empresas (Deng, 2003).

Una cuarta alternativa que eligieron algunas empresas orientadas al mercado de las nuevas tecnologías y otros productos de alta tecnología como Legend Group (Lenovo) y Huawei fue la firma de acuerdos para la importación, comer- 
cialización y reparación dentro del territorio nacional de productos tecnológicamente sofisticados, lo que les ayudó a incrementar sus conocimientos y experiencia sobre los artículos importados (Teagarden y Cai, 2008; Deng, 2009). De esta forma comenzaron a acumular conocimientos para luego lanzar al mercado sus propios productos. ${ }^{8}$

En síntesis, de acuerdo con los argumentos presentados puede concluirse que durante la primera fase de evolución de las EMN chinas la estructura oligopólica de la industria no originó economías de escala ni ahorro de costos de transacción ni generación de ventajas particulares de la firma como lo predicen las teorías convencionales debido principalmente a la intervención del gobierno (véase el cuadro 1).

El contacto con otras empresas en el extranjero (mediante licencias, joint ventures internacionales e IED) les permitió recibir transferencia de tecnología desde el extranjero para tratar de competir en una economía de mercado. De esta forma, empezaron a exportar a China activos tanto tangibles como intangibles (Wu y Chen, 2001). Es decir, mientras que, de acuerdo con las teorías generalmente aceptadas (Dunning, 1981), las EMN convencionales transfieren tecnología y conocimientos desde el país de origen hasta aquel en que se localizan sus inversiones, las EMN chinas realizaron inversiones en el extranjero para, desde ahí, transferir tecnología y conocimientos hacia China. En otras palabras, la estrategia de las firmas durante la primera fase consistió en acometer la internacionalización con la finalidad de transferir a sus empresas dos elementos básicos para su aprendizaje: tecnología industrial y know how. De esta forma comenzaron a disminuir sus desventajas en el mercado internacional.

\section{Segunda fase: crecimiento en el mercado internacional}

En la segunda fase, el ambiente de negocios adverso, el interés por ascender en la cadena de valor y la realización de diversas FyA en el extranjero marcaron la evolución de las EMN chinas.

\footnotetext{
8 Los casos de las compañías orientadas al mercado de nueva o alta tecnología suelen diferir de las EE como el Shougang Group debido a que dichas empresas fueron fundadas años después del inicio de la apertura y no se vieron sometidas a algunas de las limitantes mencionadas en esta fase, principalmente porque algunas eran privadas y otras pertenecían a institutos de investigación cuya visión estaba más orientada a los negocios que a la política (Zhou, 2008).
} 


\section{Cuadro 1}

Resumen de la conducta estratégica de las firmas chinas ante los fallos de mercado

\begin{tabular}{|c|c|c|c|}
\hline $\begin{array}{l}\text { Fallos de mercadol } \\
\text { Fases de evolución }\end{array}$ & Primera fase & Segunda Fase & Tercera fase \\
\hline Oligopolios & $\begin{array}{l}\text { Gran competencia con- } \\
\text { tra TVE y empresas pri- } \\
\text { vadas como principal } \\
\text { causa de la salida de } \\
\text { IED. }\end{array}$ & $\begin{array}{l}\text { Competencia contra } \\
\text { EMN extranjeras como } \\
\text { principal causa de la } \\
\text { salida de IED. El gobier- } \\
\text { no comienza a generar } \\
\text { programas de apoyo } \\
\text { específicos para la IED. }\end{array}$ & $\begin{array}{l}\text { Igual que en la } 2 \text { a fase, } \\
\text { más deseo de presencia } \\
\text { global como causa de } \\
\text { la IED. El gobierno fo- } \\
\text { menta la expansión en } \\
\text { el extranjero mediante } \\
\text { diversos programas. }\end{array}$ \\
\hline
\end{tabular}

Costos de transacción

Bienes diferenciados

Asimetrías de conocimiento

Economías de escala
Muy elevados debido a la fragmentación de mercados e instituciones legales subdesarrolladas. Las firmas se adaptan y buscan generar beneficios aprovechando el dual-track system.

Prácticamente no existen. Las firmas venden lo que el plan estatal establece.

No existe entre las empresas nacionales pero si con respecto a las extranjeras. Los costos son más importantes que la diferenciación y la calidad.

La organización y diversificación de las firmas y del mercado interno no permiten crecer para alcanzar economías de escala. Existe especialización en industria ligera con costos muy bajos, esto facilita la IED en países en desarrollo.
Las firmas se interesan por diferenciar productos y servicios, buscan alianzas con EMN extranjeras para lograrlo.

Grandes obstáculos a la transferencia de tecnología. La solución: realizar proyectos de nueva planta у ғун.

La organización de las firmas y las características del mercado dificultan acceder a economías de escala, la alternativa es la IED y la IED de ida y vuelta.
Las firmas (especialmente en sectores de alta tecnología) apuestan por la diferenciación de productos por medios propios o comprando marcas y patentes.

Las firmas establecen centros de I+D en diversos países.

Mejora la productividad. Las firmas localizan en el mercado interno sus actividades de producción.

\footnotetext{
Fuente: elaboración propia.
} 


\section{Ambiente de negocios adverso}

La fragmentación y el proteccionismo del mercado interno continuó generando elevados costos de transacción en ésta y la siguiente fase. La política de atracción de IED hacia el mercado interno propició que las EMN extranjeras gozaran de beneficios que afectaban a las empresas nacionales al dejarlas desprotegidas frente a la competencia. Boisot y Meyer (2008) afirman que el costo de invertir en China era tan elevado que algunas empresas habrían optado por invertir en el exterior para beneficiarse de los menores costos en otros mercados, incluso antes de estar preparadas para realizar IED. Esa actitud en contra de las empresas nacionales motivaría la IED de ida y vuelta (Boisot y Meyer, 2008) mediante la cual las firmas localizarían sus sedes y centros de investigación en ultramar y regresarían a China en calidad de empresas extranjeras para realizar sólo determinadas actividades.

\section{Interés por ascender en la cadena de valor}

En esta fase las firmas optaron por dos tipos de IED con fines muy distintos: la localizada en países en desarrollo y aquella orientada a países desarrollados.

Las inversiones en los primeros les permitieron aprovechar un mercado caracterizado por un bajo poder adquisitivo y menor desarrollo en industria ligera en la que las firmas chinas habían alcanzado ciertas ventajas, sobre todo en lo relativo a costos. Gracias a ello comenzaron a controlar dichos mercados y a exportar a los países vecinos, percatándose de que los mercados de los países en desarrollo eran relativamente fáciles de penetrar, por lo que comenzaron a invertir cada vez más en ellos. Aun así las inversiones eran pequeñas, por lo que no se beneficiaban excesivamente de las economías de escala, sobre todo al inicio de esta fase (Wu y Chen, 2001; Wang, 2002).

Sin embargo, las firmas también deseaban ascender en la cadena de valor, incrementar su calidad, mejorar sus técnicas de producción y ser más eficientes, así que comenzaron a invertir en los países desarrollados con la finalidad de acelerar su proceso de modernización. Consolidar su proceso de mejora mediante el dominio y la aplicación de los nuevos conocimientos, así como expandir sus actividades internacionales fueron sus nuevos objetivos durante esta fase. Por ello pusieron énfasis en dos puntos clave: por una parte, la mejora de procesos y el incremento de la calidad y, por otra, la adquisición de la tecnología más moderna que pudieran obtener (Teagarden y Cai, 2008). 
Para lograr lo anterior emplearon varias estrategias: las empresas orientadas al mercado de productos de alta tecnología (TCL, Huawei y Lenovo) se involucraron en la fabricación en territorio nacional de equipos especiales para grandes clientes (Teagarden y Cai, 2008) y continuaron propiciando la colaboración con otras empresas mediante joint ventures (Child y Rodrigues, 2005; Bonaglia, Goldstein y Mathews, 2006; Wu y Zhao, 2007). Las joint ventures también fueron incluidas en la estrategia de empresas industriales como las del sector de automóviles (Rui y Yip, 2008), mientras que las petroleras como CNOOC o Sinopec se decantaron por las FyA y los proyectos de nueva planta gracias al fuerte apoyo que recibían del gobierno (Eurasia Group, 2006).

Una característica distintiva entre las empresas como TCL, Huawei, Haier y Lenovo y las EE industriales es que las primeras comenzaron a invertir en I+D (Wu y Zhao, 2007; Teagarden y Cai, 2008), mientras que la estrategia de las segundas fue la de adquirir tecnología mediante FyA, sobre todo cuando (como en los casos de Nanjing Automobile Group y de Shanghai Automotive) se dieron cuenta de que las EMN con las que habían realizado joint ventures no tenían ninguna intención de proporcionarles tecnología más avanzada ${ }^{9}$ (Rui y Yip, 2008; Deng, 2009). No obstante, las joint ventures continuaron realizándose mayormente en el caso de empresas cuyo objetivo era la búsqueda de mercados. De esta manera disminuían el riesgo al entrar en mercados de gran tamaño o con significativas diferencias culturales (Wu y Chen, 2001; Cui y Jiang, 2009).

Por consiguiente, las EMN chinas siguieron utilizando joint ventures sobre todo como estrategia de entrada a nuevos mercados, pero cuando se trataba de obtener tecnología y otros activos estratégicos preferían realizar $\mathrm{FyA}^{10}$ e incluso proyectos de nueva planta. La razón subyacente en este comportamiento era el deseo de las firmas de adquirir renombre en esacala internacional y poseer marcas de prestigio, tecnología, centros de investigación, canales de distribución, etcétera, ya sea por medio de proyectos de nueva planta, como en el caso

9 Este ha sido uno de los mayores obstáculos que han tenido que enfrentar las firmas chinas: el escrutinio (sobre todo en el ámbito político) y las limitaciones que Estados Unidos y otros países de Occidente han impuesto a la transferencia de tecnología (Zhou, 2008).

${ }^{10}$ Las FyA se han realizado mayormente en países desarrollados, en operaciones que en muchas ocasiones se han visto frenadas por diversos obstáculos (como la fallida compra de Unocal por parte de CNOOC) relativos a la desconfianza que suscitan las EMN chinas y en sectores en donde hay una gran competencia, como el sector energético, la electrónica, las telecomunicaciones, los electrodomésticos, la maquinaria y los automóviles (Rui y Yip, 2008). 
de Haier en Estados Unidos, o de adquisiciones, como en el caso de Lenovo cuando adquirió IBM (Child y Rodrigues, 2005; Rui y Yip, 2008; Deng, 2009). Al mismo tiempo, las compañías chinas esperaban que esta estrategia las llevara a convertirse con el tiempo en compañías globales.

Un caso aparte es el de la internacionalización de las empresas constructoras chinas, que no pasaron por el proceso de aprendizaje descrito en la primera fase y tampoco emprendieron alianzas con empresas extranjeras. Sin embargo, gracias a sus bajos costos de producción, experiencia en proyectos complejos en gran escala dentro de su país y su especialización en infraestructura, han experimentado un gran crecimiento que les ha permitido arrebatar proyectos a las grandes constructoras internacionales (Chen y Orr, 2009).

\section{Fusiones y adquisiciones en el extranjero}

La entrada de cada vez más multinacionales en el mercado chino motivó que las firmas nacionales buscaran nuevas estrategias con las que responder a la competencia que llegaba del exterior (Rui y Yip, 2008, Teagarden y Cai, 2008). No sólo se trataba de crecer en el exterior sino de no perder su mercado interno. Infortunadamente las restricciones en la demanda, la fragmentación de los mercados y la dificultad de abastecimiento interno, por un lado, limitaban su crecimiento y, por otro, daban lugar a un exceso de producción (Buckley et al., 2008). Por tanto, una vía para deshacerse de estos problemas fue realizar las FyA (además de las joint ventures y los proyectos de nueva planta ya mencionados) que les permitieran realizar sus actividades con mayor libertad.

Además, a medida que pasaba el tiempo las firmas tomaron conciencia de que el resto de las EMN no compartirían sus tecnologías mediante joint ventures y de que sus acciones despertaban la desconfianza de la comunidad empresarial internacional. Por otra parte varias firmas chinas comenzaron a incursionar en mercados muy competitivos, como los de las telecomunicaciones, los electrodomésticos y los automóviles, por lo que la dificultad para obtener nueva tecnología aumentaba (Rui y Yip, 2008; Zhou, 2008).

Asimismo, la necesidad de contar con activos estratégicos como minas y campos petroleros propició nuevas FyA internacionales. La tendencia a realizar un número mayor de éstas coincidiría con una etapa avanzada de la segunda fase, en la que muchas firmas chinas habrían desarrollado mayor experiencia y confianza en sus operaciones internacionales por lo que preferían penetrar 
mercados mediante una empresa de propiedad total en vez de hacerlo por la vía de una joint venture, como se aprecia en el cuadro 2.

\section{Cuadro 2}

Modo de entrada de la IED China: 1991-2001

(porcentaje de las filiales extranjeras)

\begin{tabular}{lccccccccccc} 
Modo entrada & 1991 & 1992 & 1993 & 1994 & 1995 & 1996 & 1997 & 1998 & 1999 & 2000 & 2001 \\
\hline Propiedad total & 30 & 32 & 42 & 46 & 52 & 62 & 55 & 58 & 58 & 58 & 70 \\
Joint ventures & 70 & 68 & 58 & 54 & 48 & 37 & 45 & 42 & 42 & 42 & 30 \\
\hline
\end{tabular}

Fuente: Buckley et al. (2008: 734)

Varias razones dieron lugar al predominio de las empresas de propiedad total sobre las joint ventures. En primer lugar, las agencias estatales comenzaron a autorizar más inversiones financiadas totalmente con fondos chinos, a diferencia del pasado, cuando preferían joint ventures para disminuir riesgos. Muchas de estas inversiones se financiaron con créditos otorgados a países subdesarrollados (como los africanos) y se orientaron a inversiones extractivas principalmente, de acuerdo con ciertos programas de apoyo gubernamentales, lo cual se explicará en detalle cuando se hable de las instituciones como origen de las imperfecciones. En segundo lugar, en esa época la política estatal permitió que las EE tuvieran acceso a mayores recursos para financiar sus proyectos. En tercer término, las propias EE también habían desarrollado experiencia en la obtención de recursos en los mercados internacionales, por lo que podían afrontar la inversión que implica establecer una empresa de propiedad total. En cuarto lugar, la propiedad total de las subsidiarias permitía una mejor protección de los activos intangibles y evitaba la dependencia de terceros. Por último, la ola mundial de entradas a nuevos mercados internacionales mediante adquisiciones también tuvo efecto en las firmas chinas (Buckley et al., 2008).

Los avances realizados por las empresas en esta fase no impidieron que se cometieran errores. Debido a que en los consejos de administración de las firmas por lo regular ha prevalecido la idea (impulsada por la política estatal) de incrementar los activos de las EE, se efectuaron grandes inversiones que no siempre fueron productivas. Una de las razones que dio lugar al empleo ineficiente de recursos puede atribuirse a la debilidad del sistema bancario. Los fuertes apoyos económicos proporcionados por los bancos (basados en criterios políticos más que económicos) y el relajamiento del sistema de supervisión y garantías 
provocaron que se aprobaran proyectos inviables y que se mantuvieran a flote empresas improductivas (Movshuk, 2004; Naughton, 2007).

Algo parecido sucedió con algunos negocios internacionales. Con la finalidad de mejorar su reputación en escala mundial algunas empresas adquirieron activos que no resultaron rentables, como en el caso de TCL que en el año 2000 realizó una joint venture con la empresa hindú Baron Internatina Ltd. en la que invirtió 20 millones de dólares; no obstante, hubo conflictos entre ambas empresas que obligaron a TCL a retirarse de ese mercado, sólo para regresar a él en 2004 con un proyecto de nueva planta (Donghong, 2009).

En consecuencia, puede decirse que la inexperiencia en los mercados internacionales y el bajo nivel gerencial en las empresas propiciaron que algunas de las inversiones en ultramar resultaran en pérdidas (Wu y Chen, 2001).

En síntesis, durante la segunda fase de evolución las EE chinas recurrieron a la IED como una forma de enfrentar los fallos de su mercado (véase el cuadro 1). Al mismo tiempo, durante esta fase de evolución, las firmas dedicaron sus esfuerzos a alcanzar tres objetivos principales: en primer lugar, adquirir tecnología de vanguardia, marcas y patentes y otros activos estratégicos, mayormente mediante FyA y proyectos de nueva planta ; en segundo lugar, desarrollar productos y procesos más sofisticados y de mayor contenido tecnológico, haciendo énfasis en la calidad, y, en tercer lugar, ampliar sus mercados y labrarse un nombre para mejorar su reputación en escala mundial y evitar ser discriminadas por su procedencia, dejando atrás su pasado reciente.

La mayoría de las EMN chinas está actualmente en esta fase y continúan su proceso de aprendizaje y mejora. Sin duda han realizado un gran avance (tomando en cuenta sus orígenes) y algunas son líderes entre las EMN de países en desarrollo. Sin embargo, su presencia es regional, no global, pues aún no han impulsado suficientemente sus productos y procesos, su estructura organizacional, su conocimiento del mercado y su personal. Además, algunas firmas aún dependen excesivamente de los apoyos del gobierno. En consecuencia, todavía no han desarrollado suficientes ventajas que les permitan enfrentar con éxito a las EMN de los países desarrollados.

\section{Tercera fase: globalización}

Uno de los sectores más abiertos dentro del mercado chino es el de las nuevas tecnologías de la información y la comunicación; de ahí que las grandes multinacionales de dicho sector tengan una importante presencia en el país. Esto ha 
afectado el desempeño de las empresas nacionales que se han visto forzadas a emprender acciones para defender sus mercados (Rui y Yip, 2008; Zhou, 2008). En otras palabras, una vez iniciada la internacionalización, no había marcha atrás: era necesario hacer frente a las grandes multinacionales de los países desarrollados tanto dentro como fuera de China para evitar ser eliminadas por la competencia y lograr la competitividad en escala global.

Esta batalla la han librado principalmente empresas como Lenovo (ordenadores), Haier (electrodomésticos) y Huawei (telecomunicaciones). Sus marcas poseen reconocimiento en el exterior y han desarrollado considerablemente su mercado internacional (Bonaglia, Goldstein y Mathews 2006; Rui y Yip, 2008). Para lograr sus objetivos las firmas han emprendido diversas estrategias que las están conduciendo a una posición de liderazgo mundial.

En primer lugar, puede decirse que la base de su estrategia es la innovación y el desarrollo de productos, por lo que han invertido en centros de I+D localizados dentro y fuera de China. Algunos de los centros de investigación han sido parte de los activos adquiridos mediante FyA. En segundo lugar, poseen una cadena de suministro y producción de alta calidad que les aseguran los mejores componentes para sus productos. En este aspecto, han establecido sus plantas de ensamblaje en territorio nacional debido a que sus proveedores también están localizados ahí, lo que facilita los intercambios y el trabajo en equipo con aquéllos. En otras palabras, las compañías en esta fase han aprendido a aprovechar las sinergias generadas por el crecimiento del sector de las nuevas tecnologías y su propia capacidad exportadora. En tercer lugar, se han preocupado por conocer el mercado, los gustos y las expectativas de los consumidores. En cuarto lugar, el establecimiento de diversas sedes en el mundo ha ido acompañado del desarrollo de personal en dos aspectos clave: tecnología de productos y visión global de negocios (Teagarden y Cai, 2008; Zhou, 2008).

Respecto a los fallos del mercado (véase el cuadro 1), las firmas en esta etapa los afrontan de forma similar a lo que predicen las teorías convencionales. No obstante, como el mercado interno todavía no ha sido liberalizado totalmente, en la actualidad continúan enfrentándose a costos de transacción altos y a la intervención del gobierno en sus decisiones, como lo demuestran los procesos de aprobación descritos por Pamlin y Baijin (2007). Simultáneamente existen apoyos extraordinarios para impulsar la expansión de las empresas que están en la etapa de globalización y algunas que transitan entre la segunda fase y ésta, las cuales se beneficiaron del avance de la economía china, que registró tasas de 
crecimiento por encima de 8\% entre 1995 y 2011 (FMI, 2013), lo que permitió al país contar con un nivel histórico de reservas en moneda extranjera (3.1 billones de dólares en 2011) que, a su vez, contribuyó a crear un robusto fondo de inversión para la IED ${ }^{11}$ (Gallagher, Irwin y Koleski, 2012; Downs, 2011). Conjuntamente, el gobierno chino modificó su legislación con la finalidad de apoyar fusiones y adquisiciones en el extranjero reduciendo los requisitos para evaluación de préstamos en el exterior y permitiendo a las empresas chinas utilizar reservas de divisas propias, así como reservas oficiales para hacer préstamos a sus filiales en el extranjero. Aunado a la anterior, la crisis financiera de 2008 creó condiciones favorables para incrementar el número de FyA chinas en el mundo como puede verse en el cuadro 3 (Cha, 2009).

Cuadro 3

Número de fusiones y adquisiciones internacionales chinas

\begin{tabular}{ccccccccccc}
\hline 2001 & 2002 & 2003 & 2004 & 2005 & 2006 & 2007 & 2008 & 2009 & 2010 & $2011^{*}$ \\
\hline 69 & 66 & 60 & 81 & 93 & 125 & 181 & 180 & 216 & 252 & 151 \\
\hline
\end{tabular}

Nota: * Cifras de UnCTAD (2012).

Fuente: Baird (2011).

Así, entre 2008 y 2012 el Geely Holding Group compró a Ford la empresa Volvo (localizada en Suecia), así como la inglesa Manganese Bronze; ChemChina adquirió la noruega Elkem por medio de su filial China National Bluestar Group; la holandesa InterGen fue adquirida por el China Huaneng Group; la estadounidense AIG acordó vender $80 \%$ de la International Lease Finance Corporation a un grupo de inversionistas chinos y el Tempo International Group Limited compró a General Motors la empresa Nexteer Automotive (Baird, 2011; Egan, 2012). De esta forma puede decirse que la etapa de globalización en diversas EMN chinas se vio estimulada debido a dos situaciones principalmente: en primer lugar, al apoyo del gobierno mediante medidas legislativas y financieras y, en segundo lugar, a las condiciones creadas por la crisis económica.

El análisis de las tres fases muestra cómo las firmas chinas comenzaron a realizar inversiones en el extranjero a pesar de su producción ineficiente, au-

${ }^{11}$ Por ejemplo, la China Investment Corporation (CIC) se estableció en septiembre de 2007como un fondo soberano de inversión, mediante un financiamiento inicial de 200 mil millones de dólares de las reservas del Banco Popular de China. Así, en 2012, la CIC estableció una empresa junto con Global Logistic Properties, de Singapur, y Canada Pension Plan Investment Board and Government con el objeto de construir la mayor plataforma de logística en Brasil (Baird, 2011). 
sencia de productos diferenciados y de I+D, así como de sus elevados costos de transacción. Lejos de desalentar su internacionalización, estas carencias sirvieron de acicate a su propósito, por lo que se dedicaron a aminorar la brecha entre ellas y el resto de EMN utilizando la IED como estrategia principal. Haciendo un recuento de lo expuesto hasta ahora, es posible afirmar que lo que realmente marca la diferencia entre la IED china y la de otros países no es únicamente la conducta atípica mostrada por sus EMN ante las imperfecciones de mercado (haber salido al extranjero en las condiciones descritas y luego escalar en la cadena de valor gracias a las FyA y a los proyectos de nueva planta), sino también el impacto que causan en la conducta de las EMN las imperfecciones provocadas por las instituciones chinas, que por una parte alientan la emisión de IED y por otra regulan y controlan la misma. Como puede apreciarse en el cuadro 1, el origen de muchos de los obstáculos así como de las ayudas extraordinarias para su expansión está en las instituciones. Es este impacto el que no ha sido incluido para su estudio en la teoría económica convencional, pero que dadas las circunstancias es imprescindible analizar, lo que se hará a continuación.

\section{LAS INSTITUCIONES COMO ORIGEN DE LAS IMPERFECCIONES}

Considerando la información analizada hasta ahora, queda en evidencia que los principales fallos a los que se han enfrentado las firmas chinas en su mercado interno no han sido sólo aquellos provocados por el mercado propiamente dicho sino por las instituciones, lo que ha modificado las condiciones de su propio mercado creando las alteraciones que han afectado el desempeño de las empresas.

Los fallos promovidos por las instituciones tienen un carácter dual: en primer lugar, los factores limitantes que por el excesivo control han propiciado la expulsión de capitales chinos por la vía de la IED y, en segundo lugar, los factores motivantes que han impulsado, mediante diversos mecanismos de ayuda, las inversiones transfronterizas. La presencia de los factores limitantes se aprecian principalmente durante la primera y parte de la segunda fase de desarrollo, comenzando a remitir sobre todo a partir de la implementación de la política de "salida al exterior" 12 (sin desaparecer del todo) que es cuando los factores motivantes cobran fuerza.

${ }^{12}$ Esta política se anunció formalmente en octubre del año 2000 como parte del X Plan Quinquenal chino 2001-2005 (Sauvant, 2005). 


\section{Factores de expulsión}

A la luz de los argumentos expuestos hasta ahora puede decirse que entre las limitantes principales a las que se enfrentan las firmas en su mercado interno sobresalen los que a continuación se describen.

Mercados fragmentados, proteccionismo regional y excesivos niveles administrativos

Como se explicó previamente, todo ello limita el acceso a los mercados internos e impide aprovechar las economías de escala. A esto hay que añadir que los diversos niveles de la administración pública impiden cruzar los límites jurisdiccionales, lo que frena las actividades que requieren una organización horizontal, como los servicios de transporte multimodal, los cuales funcionan con base en la cooperación entre diferentes proveedores de los mismos y la cual se ve frenada por las limitaciones que cada jurisdicción impone en su territorio.

Lo anterior complica las transacciones, alarga los tiempos y eleva los costos, sobre todo en las provincias interiores, donde el desarrollo del transporte es menor. En consecuencia, los altos costos de transporte asociados a la cadena de suministro socavan la competitividad de las firmas en el mercado nacional.

\section{Debilidad institucional y sistema legislativo dual}

La debilidad institucional se debe a que la legislación vigente no brinda una protección adecuada de los derechos de propiedad intelectual. A su vez, esta circunstancia origina una baja motivación para el desarrollo de I+D. Los costos de transacción se elevan debido al sistema legal débil e incierto que prevalece. $\mathrm{Al}$ mismo tiempo, existe discriminación hacia las empresas nacionales debido a una legislación dual que privilegia a las extranjeras, dándoles mayor libertad de acción ${ }^{13}$ pero que constriñe a las empresas chinas, según lo expuesto en el apartado anterior.

\footnotetext{
${ }^{13}$ Un ejemplo de ello son la Law on Sino-Foreign Equity Joint Ventures de 1979, la Wholly Foreign-Owned Enterprise Law de 1986 y la Sino-Foreign Cooperative Joint Venture Law de 1988 con las que se inició un sistema de beneficios para las empresas extranjeras.
} 


\section{Acceso condicionado al financiamiento}

La existencia de documentos como los Guiding Directories of Target Nations and Industries for OFDI, que enlistan una serie de países e industrias en las que se favorece la inversión con preferencias tributarias y administrativas; de instituciones como el Fondo de Desarrollo China-África, que proporciona fondos para la IED, la promoción de cierto tipo de exportaciones y algunos sectores, así como la preferencia por un tipo de propiedad (EE por encima de otro tipo de empresas) han propiciado que el financiamiento destinado a proyectos de IED esté muy condicionado.

De la misma forma, la existencia de bancos manejados internamente (como el Huaxia Bank descrito previamente) contribuyó a generar ventajas para unas empresas pero alteró las condiciones del mercado de dinero para otras que no tenían las mismas posibilidades. Por ello, algunas empresas, sobre todo durante la primera fase, invirtieron en Hong Kong y buscaron financiamiento en el extranjero (Zhang, 2008).

\section{Numerosos procesos de aprobación}

Antes de invertir en el extranjero las firmas deben pasar por un proceso de aprobación que puede durar incluso meses (Pamlin y Baijin, 2007; Buckley et al., 2008; Chao y Ji, 2008). A pesar de que los controles han disminuido con el tiempo y que el número de procedimientos se ha reducido, aún persisten una serie de prácticas que obstaculizan la libertad de las empresas, incluso en el exterior, como en el caso de las constructoras que antes de presentarse a concurso en un país extranjero deben contar con la aprobación de la consejería económica china en el país de destino y de la China International Contractors Association (Chen y Orr, 2009).

A la luz de lo expuesto en este apartado, puede concluirse que en gran medida las empresas chinas han sido expelidas del territorio nacional debido a los fallos que directa o indirectamente han generado las instituciones. En otras palabras, tales empresas han buscado localizar sus actividades en el exterior intentando encontrar mercados mejor organizados, que faciliten sus actividades y que las liberen de los fallos institucionales a los que se ven sometidas en su país de origen. Estas observaciones coinciden con lo expuesto por Brewer (1993), quien afirma que las políticas de gobierno generan imperfecciones de 
mercado que hacen más atractivo para una firma invertir en el extranjero que realizar exportaciones. Dicho autor identifica como políticas que aumentan las imperfecciones de mercado algunos de los elementos expuestos en esta investigación: los subsidios a la IED, los controles a la salida de capitales, los controles de precios y los subsidios a las exportaciones.

\section{Factores de motivación}

Entre los factores de motivación generados por el gobierno chino sobresalen tres: el apoyo institucional y diplomático, el apoyo financiero y los privilegios varios.

\section{Apoyo institucional y diplomático}

Uno de los mecanismos de apoyo que destaca es un esquema de control y ayuda in situ (denominado en esta investigación esquema CHINCA) que está relacionado con la construcción y ha sido aplicado en los países africanos principalmente. La Asociación Internacional de Contratistas Chinos (China International Contractors Association, CHINCA), las consejerías económicas de cada embajada y el Exim Bank actúan de manera conjunta para favorecer la contratación de firmas chinas en proyectos de infraestructura en el extranjero (Chen y Orr, 2009).

Un segundo mecanismo, que complementa el esquema CHINCA, es el modelo Angola (conocido con ese nombre por haberse aplicado en primer lugar en dicho país), que ejemplifica el apoyo institucional y diplomático para propiciar el trueque de productos (básicamente materias primas) por préstamos provenientes de los bancos chinos (Foster et al., 2008). Así, con el esquema CHINCA se otorgan préstamos para la construcción de infraestructura y mediante el modelo Angola algunos préstamos se pagan en especie, petróleo principalmente. Otros mecanismos de ayuda son los mencionados Guiding Directories of Target Nations and Industries for OFDI.

\section{Apoyo financiero}

El gobierno chino genera imperfecciones en el mercado financiero por cuatro vías principales: a) los préstamos especiales; $b$ ) la operación de bancos internos por parte de algunos conglomerados; c) el trabajo conjunto entre bancos, organismos del gobierno y embajadas para conformar esquemas como el CHINCA y el 
modelo Angola, $y d$ l) las recientes modificaciones a la ley que permiten a las EMN utilizar reservas de divisas propias y del gobierno para FyA internacionales.

En el primer caso, los Chinese Government Concessional Loans y los Preferential Export Buyer's Credits otorgados por el Exim Bank de China apoyan la IED promoviendo la cooperación económica entre países, obligando a la contratación de empresas chinas y, en caso de ser necesario, a la compra de bienes procedentes del país asiático.

En el segundo caso, el Consejo de Estado, por ejemplo, aprobó la transferencia de la China Investment and Trust Corporation for Foreign Economic Cooperation and Trade (FOTIC) al Grupo Sinochem, para que funcionara como banco interno del grupo; mientras que al Shougang Group se le permitió crear el propio (Huaxia Bank), lo que garantizaría que su presupuesto no tuviera limitaciones (Buckley et al., 2007). Con el tiempo tanto la FOTIC ${ }^{14}$ como el Huaxia ${ }^{15}$ Bank fueron restructurados, lo que ha dado mayor transparencia a sus operaciones y ha evitado que las firmas hagan mal uso de los recursos, como en el caso del Shougang Group mencionado.

En el tercer caso, un buen ejemplo es el modelo Angola y el esquema CHINCA explicados previamente, que alteran las condiciones del mercado al privilegiar la entrega de recursos a ciertas empresas y a algunos sectores (Foster et al., 2008; Chen y Orr, 2009).

En el cuarto caso, en diciembre de 2008 la Comisión Reguladora de la Banca de China emitió las Directrices para la Gestión de Riesgos de los Préstamos Otorgados por Bancos Comerciales para Fusiones y Adquisiciones (en adelante las directrices) para permitir a los bancos chinos otorgar préstamos para ese propósito en el extranjero, reducir los requisitos para la evaluación de préstamos en el exterior, expandir las fuentes de fondos destinados a éstos y simplificar los procedimientos de verificación y envío de remesas. Las directrices fueron emitidas para incrementar el apoyo bancario respecto a las FyA internacionales

${ }^{14}$ La FOTIC se creó en 1987 y se le incorporó a Sinochem en 1994 al fusionarse con una empresa financiera propiedad de la firma. En 2002 se le otorgó una nueva licencia para que realizara actividades financieras de acuerdo con las Regulations on Trust Enterprises y a las Regulations on Collective Fund Trust Scheme for Trust Enterprises de la Comisión Reguladora de la Banca de China, quedando bajo la supervisión de este organismo. Actualmente la FOTIC continúa siendo una empresa subsidiaria de Sinochem (Sinochem, 2012).

15 Tras varias reestructuraciones el Huaxia Bank comenzó a cotizar en bolsa en 2003. Parte de las acciones fueron adquiridas por el Deutsche Bank en 2006 y 2008, pero el Shougang Group aún conserva una participación mayoritaria (Deutsche Bank, 2010). 
aprovechando la coyuntura creada por la crisis que puso al borde de la quiebra a muchas empresas occidentales y abarató sus activos. Asimismo, en junio de 2009 la Administración Estatal de Divisas publicó el Comunicado sobre Cuestiones Relativas a la Administración de Divisas en Préstamos en el Extranjero por Empresas Nacionales (en adelante, el comunicado). Debido a que la crisis financiera dificultó que las filiales de las empresas chinas obtuvieran financiamiento externo, mediante el comunicado se simplificó el proceso para la financiar a las filiales mediante la reducción de las estipulaciones de calificación para los préstamos en el exterior, la ampliación de las fuentes de fondos para préstamos y la disminución de los procedimientos de verificación y envío de remesas para los préstamos (Benesch et al., 2010).

\section{Privilegios varios}

Entre los privilegios que gozan las empresas que invierten en el extranjero destacan el acceso preferente a materias primas y otros recursos, la promoción de exportaciones orientadas a la IED (es decir, créditos para la venta externa de maquinaria y equipo con el fin de instalar unidades fabriles en el extranjero), menores impuestos para empresas exportadoras y sectores privilegiados (Nolan y Yeung, 2001b; Buckley et al., 2007; Deng, 2007).

En síntesis, las instituciones y organismos mencionados han modificado favorablemente las condiciones de las firmas chinas al facilitar su salida al extranjero, especialmente a partir de la política de "salida al exterior".

\section{LA CONDUCTA DE LAS EMN CHINAS ANTE LOS FALLOS PROVOCADOS POR LAS INSTITUCIONES}

Tanto los factores de motivación como los de expulsión han suscitado ciertas conductas en las firmas chinas. Habiendo estado presentes desde el principio de la apertura, se describirán en primer lugar los factores de expulsión y posteriormente se hará lo propio con los de motivación.

Considerando la información analizada hasta ahora se puede aseverar que las firmas han mostrado a lo largo del tiempo una conducta estratégica para tratar de minimizar el efecto negativo de los factores de expulsión.

En primer lugar, las restricciones y los permisos impuestos a la IED han provocado la salida ilegal de capitales (Ding, 2000; Gunter, 1996). En segundo 
lugar, también han propiciado que, una vez recibida la primera autorización, las firmas no repatrien las ganancias y que en vez de ello las reinviertan en el extranjero sin notificar al Ministerio de Comercio (Shan, 1989). En tercer lugar, el proteccionismo, los excesivos niveles de la administración pública y la intervención del gobierno en las decisiones de las firmas desalentaron la generación de ventajas I y $\mathrm{O}$, lo que las colocaba en desventaja frente al resto de multinacionales; así que tuvieron que realizar IED para generar tales ventajas y poder sobrevivir, como se explicó anteriormente. En cuarto lugar, los fallos en el mercado financiero (financiamientos condicionados, permisos, privilegios a ciertas empresas) propiciaron la salida de las empresas para buscar financiamiento en el extranjero, como en el caso de las inversiones hechas en Hong Kong para cotizar en la bolsa (Zhang, 2008).

En cuanto a los fallos provocados por los factores de motivación también existen algunas conductas relacionadas con ellos.

Primero, el apoyo institucional y diplomático ha facilitado la salida de las firmas, impulsándolas a realizar IED en países que de otra forma no habrían sido elegidos, o bien por no reunir condiciones de mercado óptimas (países con riesgo político), o bien por tratarse de firmas relativamente nuevas con poca experiencia en mercados desarrollados como lo describen Buckley et al. (2007). Igualmente, la reciente expansión mediante FyA en países desarrollados se incrementó en parte gracias al apoyo del gobierno.

Segundo, los fallos propiciados por el financiamiento y otras ayudas descritas han generado en las firmas una conducta altamente inusual en las EMN convencionales: elmenosprecio hacia el riesgo cuando invierten. Para explicar esta situación es necesario acudir al estudio que Buckley et al. (2007) publicaron con respecto a los determinantes de la IED china. Uno de los elementos estudiados fue el riesgo político, que fue medido econométricamente utilizando como variable dependiente el total de IED aprobada por la Administración Estatal de Divisas mientras que como variable independiente emplearon el riesgo político medido con base en la International Country Risk Guide.

La investigación planteó como hipótesis que la IED china estaría negativamente asociada a niveles elevados de riesgo en el país de destino. Sin embargo, de forma sorprendente, los resultados de la medición mostraron que había una creciente relación entre riesgo e IED pero contraria a la esperada. Es decir, a mayor estabilidad política, menor grado de IED.

Atrás analizar posibles explicaciones, los investigadores llegaron a la conclusión de que las imperfecciones del mercado financiero y los factores institucio- 
nales habían generado una actitud de menosprecio hacia el riesgo; es decir, los inversionistas chinos parecían percibir el riesgo de modo muy diferente que los occidentales pues se sentían arropados por sus instituciones y sus préstamos baratos. Otra posible explicación a esta conducta sería que parte de ese menosprecio al riesgo también se debiera a la falta de experiencia de muchas de sus empresas en los mercados internacionales, sobre todo en las primeras etapas. Cuando Cui y Jiang (2009) hicieron una medición similar sobre la IED china encontraron resultados semejantes a los de Buckley et al. (2007).

\section{Conclusiones}

Como está previsto en las teorías convencionales, en el mercado interno chino las firmas formaron oligopolios con gran poder de mercado que competían contra otras firmas también de gran tamaño y que, dado que algunas de ellas establecieron joint ventures con EMN extranjeras, poseían conocimientos comparativamente más avanzados y podían producir bienes diferenciados. Sin embargo, al contrario de lo previsto por las teorías convencionales, la estructura oligopólica de la industria no necesariamente llevaba a economías de escala, ni al ahorro de costos de transacción, ni al desarrollo de asimetrías de conocimiento y bienes diferenciados, básicamente por razones institucionales.

Las razones institucionales por las que las firmas chinas no se beneficiaron de las imperfecciones de mercado, principalmente en la primera y parte de la segunda fase de evolución, tienen que ver con que la internalización de actividades no fue producto de una toma de decisiones racional y eficiente sino que obedeció a decisiones políticas, entre ellas la anexión de negocios periféricos a las grandes EE con el fin de conservar los empleos de los trabajadores. El análisis también reveló que en el desarrollo de las firmas chinas cada una de las fases de evolución (fase de aprendizaje, crecimiento en el mercado internacional y globalización) estuvieron marcadas no solamente por los cambios propios de la firmas en su desarrollo de empresas nacionales a globales, sino sobre todo por los cambios institucionales generados desde los diferentes niveles de gobierno. Como consecuencia de tales mudanzas, el proceso de desarrollo de las firmas, que permite la creación e incremento de ventajas oLI, se alteró, dando lugar a conductas diferenciadas de aquellas mostradas por las EMN convencionales.

La primera conducta diferenciada es la realización de IED sin haber generado ventajas $\mathrm{O}$ e I, conducta propiciada por lo que en este trabajo se llamaron 
factores de expulsión (mercados fragmentados, proteccionismo regional y excesivos niveles administrativos; debilidad institucional y sistema legislativo dual; acceso condicionado al financiamiento y numerosos procesos de aprobación), factores que estuvieron presentes primordialmente en la primera fase y aunque se relajaron a mediados de la segunda fase, no desaparecieron del todo.

La segunda conducta diferenciada es la adquisición (en vez de la generación interna, como lo establece el paradigma ouI) de tecnología y know how por medio de: a) joint ventures con EMN extranjeras tanto dentro de su mercado interno como fuera de él (primordialmente en la fase de aprendizaje y de crecimiento en el mercado internacional); b) FyA internacionales (fundamentalmente en las fases de crecimiento en el mercado internacional y de globalización), y c) proyectos de nueva planta, primordialmente en países desarrollados.

Una tercera conducta diferenciada que se desprende de la anterior es que, debido a su necesidad de tecnología y know how, varias firmas pasaron por alto algunas de las etapas de internacionalización (concretamente la fase exportadora y establecimiento de oficinas de ventas en el extranjero) transitando directamente a la etapa de proyectos de nueva planta productivos.

Un diferencia más (la cuarta) es que las firmas no acometieron la IED como una actividad que les permitiría expandir sus dominios como predicen las teorías convencionales, sino que la asumieron como: a) una forma de liberarse de los controles del gobierno y acceder al financiamiento externo o b) el camino para conseguir los privilegios de los que gozan las EMN extranjeras en territorio chino, realizando IED de ida y vuelta. Esta conducta está presente en todas las fases pero tiende a disminuir con la llegada de la política de "salida al exterior" debido a que los factores de expulsión disminuyen.

Un caso aparte es el que representan las empresas constructoras y las firmas fabricantes de electrodomésticos y productos relacionados con las nuevas tecnologías, como Huawei, Haier y Lenovo. Como se explicó en su momento, las empresas constructoras chinas generaron ventajas de propiedad en un periodo previo a su internacionalización debido a su experiencia en grandes proyectos de construcción de bajo costo y en condiciones geográficas adversas, lo que permitió que, cuando iniciaron su internacionalización, pudieran competir sin grandes dificultades con otras constructoras en el mercado internacional, ganando desde entonces en experiencia y número de proyectos realizados.

La historia de empresas en sectores con un uso medio y alto de tecnología (la mayoría de ellas en una fase temprana de globalización) difiere de las construc- 
toras y de la mayoría de las EE, en el sentido de que varias de ellas no nacieron en el periodo previo a la apertura, sino posteriormente, como Haier y Lenovo (en 1984) y Huawei (en 1988). Además, previamente a su internacionalización pudieron adquirir experiencia ocupándose del servicio de mantenimiento y reparación de electrodomésticos, ordenadores y otros productos de alta tecnología; más tarde incluso adquirieron licencias para elaborar dichos productos en el mercado interno, y posteriormente invirtieron en sus propios centros de I+D tanto dentro como fuera del país, lo que les reportó mayores conocimientos. Todo ello les permitió acrecentar sus ventajas rápidamente, transitar de una fase a otra de desarrollo en menor tiempo y mostrar un progreso semejante al de las EMN convencionales. Por tanto, es posible que algunas de las conclusiones vertidas en este trabajo expliquen mejor la IED de las EE de otras industrias que la IED de las firmas de este particular sector, sin que ello implique que las empresas de electrodomésticos y tecnologías de la información no hayan recibido influencia de los aspectos institucionales considerados en esta investigación. Cabe añadir que algunas investigaciones sobre las EMN chinas (Sauvant, 2005; Bonaglia, Goldstein y Mathews, 2006; Accenture, 2008) han establecido sus observaciones fundamentalmente a partir de las empresas descritas en este párrafo, por lo que no es extraño que sus autores consideren que las firmas han basado su internacionalización en el desarrollo de ventajas al estilo de las teorías convencionales.

Esta investigación demuestra que a los fallos de mercado o determinantes convencionales hay que añadir aquellos que han sido originados por el gobierno (factores de expulsión y motivación) para comprender la conducta de las firmas y, por ende, el perfil de la IED china. Debido al gran peso de ambos factores en el ambiente de negocios chino y a la información expuesta, es evidente que los mismos han generado fallos de tal magnitud que probablemente han pesado más que los fallos convencionales de mercado al momento de decidir realizar inversiones en el extranjero. Por último, especial consideración merecen las empresas que, en los últimos años, han adquirido activos estratégicos en Estados Unidos y Europa marcando una tendencia en las FyA chinas que merece ser investigada en el futuro.

\section{REFERENCIAS}

Accenture, 2008. The Rise of the Emerging Market Multinational. [en línea] Accenture Institute for High Performance. Disponible en: < http://www.accenture.com/us-en/ 
research/institute-high-performance/Pages/insight-multi-polar-world-researchfindings-summary.aspx $>$ [Consultado el 22 de enero de 2012].

Baird (Robert W. Baird \& Co.), 2011. China M\&A Market, Mid-Year 2011, Overview of Economic and M\&A Activity. [en línea] Disponible en: <http://www.geasiapacifico. org/documents/China\%20MA\%202011\%20Inversion\%20y\%20Mercados.pdf> [Consultado el 29 de mayo de 2013].

Benesch, Friedlander, Coplan \& Aronoff LLP, 2010. China Insights. [en línea] Benesch, Friedlander, Coplan \& Aronoff LLP Publication. Disponible en: <http://www. beneschlaw.com/Files/Publication/58b1c8f1-d679-4051-b50f-4631d1cac3b4/ Presentation/PublicationAttachment/309b0f83-98ba-4ee8-b03f-46526897d787/ January_2010.pdf $>$ [Consultado el 28 de mayo de 2013].

Boisot, M. y Meyer, M. W., 2008. Which Way Through the Open Door? Reflections on the internationalization of Chinese firms. Management and Organization Review, 4(3), pp. 349-65.

Bonaglia, F., Goldstein, A. y Mathews, J., 2006. Accelerated Internationalisation by Emerging Multinationals: The case of white goods sector. [en línea] Research Papers in Economics. Disponible en: <http://mpra.ub.uni-muenchen.de/1485/> [Consultado el 18 April 2012].

Brewer, T.L., 1993. Government Policies, Market Imperfections, and Foreign Direct Investment. Journal of International Business Studies, 24(1), pp. 101-20.

Buckley, P.J. y Casson, M., 1976. The Future of the Multinational Enterprise. Houndmills: Palgrave MacMillan.

Buckley, P.J.; Clegg, L.J.; Cross, A.R.; Liu, X.; Voss, H. y Zheng, P., 2007. The Determinants of Chinese Outward Foreign Direct Investment. Journal of International Business Studies, 38(4), pp. 499-518.

Buckley, P.J.; Cross, A.R.; Tan, H.; Xin, L. y Voss, H., 2008. Historic and Emergent Trends in Chinese Outward Direct Investment. Management International Review, 48 (6), pp. 715-47.

Coase, R.H., 1937. The Nature of the Firm. Economica, 4 (16), pp. 386-405.

Cui, L. y Jiang, F., 2009. Ownership Decisions in Chinese Outward FDI: An integrated research framework and research agenda. Asian Business \& Management, 8(3), pp. 301-24.

Cha, A.E., 2009. China Gains Key Assets in Spate of Purchases. The Washington Post. [en línea] 17 de marzo. Disponible en: < http:/ / articles.washingtonpost.com/200903-17/world/36801426_1_outbound-investment-china-national-petroleum-chinese-companies $>$.

Chao, H. y Ji, R., 2008. Obtaining Funding and Approval for Chinese Outbound Investments. [pdf] Beijing: O’Melveny \& Myers LLP. Disponible en: <http://www.omm.com/ newsroom/publication.aspx?pub=619> [Consultado el 7 de julio de 2012.] 
Chen, C. y Orr, R.J., 2009. Chinese Contractors in Africa: Home government support, coordination mechanisms and market entry strategies. Journal of Construction Engineering and Management, 135(11), pp. 1201-10.

Child, J. y Rodrigues, S.B., 2005. The Internationalization of Chinese Firms: A case for theoretical extension? Management and Organization Review, 1(3), pp. 381-410.

Demsetz, H., 1967. Toward a Theory of Property Rights. American Economic Review, 57(2), pp. 347-59.

Deng, P., 2003. Foreign Direct Investment by Transnationals from Emerging Countries: The case of China. Journal of Leadership and Organizational Studies, 10, pp. 113-24.

Deng, P., 2007. Investing for strategic resources and its rationale: The case of outward FDI from Chinese companies. Business Horizons, 50(1), pp. 71-81.

Deng, P., 2009. Why do Chinese Firms Tend to Acquire Strategic Assets in International Expansion? Journal of World Business, 44(1), pp. 74-84.

Deutsche Bank, 2010. Deutsche Bank History. [en línea] Disponible en: <http://www. db.com/hongkong/en/content/history.html> [Consultado el 7 de julio de 2012].

Ding, X. L., 2000. Informal Privatization through Internationalization: The rise of nomenklatura capitalism in China's offshore businesses. British Journal of Political Science, 30(1), pp. 121-46.

Donghong, L., 2009. Innovation and Knowledge Transfer in Chinese Multinationals. En: Larçon, J.P. ed. Chinese Multinationals. Singapur: World Scientific, pp.151-91.

Downs, E., 2011. Inside China, Inc: China Development Bank's Cross-Border Energy Deals. [en línea] Brookings Institution, John L. Thornton China Center Monograph Series no. 3, Disponible en: <http://www.brookings.edu/ /media/Files/rc/papers/2011/0321_china_energy_downs/0321_china_energy_downs.pdf $>$ [Consultado el 28 de mayo de 2013].

Dunning, J.H., 1981. International Production and the Multinational Enterprise. Londres: George Allen \& Unwin.

Dunning, J.H., 1988. The Eclectic Paradigm of International Production: A restatement and some possible extensions. Journal of International Business Studies, 19(1), pp.1-31.

Dunning, J.H.,1993. Multinational Enterprises and the Global Economy. Berkshire: AdisonWesley.

Egan, M., 2012. AIg Inks \$4.23B Deal to Sell ILFC to Chinese Group. [en línea] Fox Business, 10 de diciembre. Disponible en: <http://www.foxbusiness.com/ industries / 2012/12/10/aig-inks-423b-deal-to-sell-lfc-to-chinese-group/ \#ixzz2LeZDa7cp> [Consultado el 28 de mayo de 2013].

Eurasia Group, 2006. China's Overseas Investments in Oil and Gas Production. [en línea] Nueva York: Eurasia Group. Disponible en: <http://www.uscc.gov/researchpapers/2006/oil_gas.pdf> [Consultado el 28 de junio de 2012]. 
FMI (Fondo Monetario Internacional), 2013. World Economic Outlook Database. [en línea] Disponible en: <http://www.imf.org/external/pubs/ft/weo/2013/01/weodata/index.aspx $>$ [Consultado el 28 de mayo de 2013].

Foster, V., Butterfield, W., Chen, C. y Pushak, N., 2008. Building Bridges: China's growing role as infrastructure financier for Sub-Saharan Africa. [en línea] Washington, D.C.: The World Bank. Disponible en: <http:// siteresources.worldbank.org/INTAFRICA/ Resources/Building_Bridges.pdf $>$ [Consultado el 28 de junio de 2012].

Gallagher, K., Irwin, A. y Koleski, K., 2012. The New Banks in Town: Chinese Finance in Latin America. Washington, DC: Inter-American Dialogue Report, febrero.

Giddy, I.H. y Young, S., 1982. Conventional Theory and Unconventional Multinationals: Do new forms of multinational enterprise require new theories? En: Rugman, A.M., ed. 1982, New Theories of the Multinational Enterprise. Londres: Croom Helm, pp. 55-78.

Gunter, F.R., 1996. Capital Flight from China: 1984-1994. China Economic Review, 7(1), pp. 77-96.

Heenan, D.A. y Keegan, W.J., 1979. Rise of Third World Multinationals. Harvard Business Review, 57(1), pp. 101-9.

Hymer, S.H., 1976. The International Operations of National Firms: A study of direct foreign investment. Cambridge, Mass.: The MIT Press.

Jones, L.P., 1975. Public Enterprise and Economic Development: The Korean case. Seúl: Korean Development Institute.

Kantarelis, D., 2007. Theories of the Firm. Buckinghamshire: Inderscience Enterprises, Landry, P., 2008. How Weak Institutions can Produce Strong Regimes: Patents, lawyers and the improbable creation of an intellectual property regime in China (1985-2007). [en línea] New Haven, CT: Yale MacMillan Center. Disponible en: <http://yale.edu/macmillan/ruleoflaw/papers/PATENT_PAPER_FINAL.pdf $>$ [Consultado el 28 de julio de 2012].

Lall, S.,1983. The Rise of Multinationals from the Third World. Third World Quarterly, 5(3), pp. 618-26.

Lecraw, D. J., 1983. Performance of Transnational Corporations in Less Developed Countries. Journal of International Business Studies, 14(1), pp. 15-33.

Movshuk, O., 2004. Restructuring, Productivity and Technical Efficiency in China's Iron and Steel Industry, 1988-2000. Journal of Asian Economics, 15(1), pp. 135-51.

Naughton, B., 2007. The Chinese economy: Transitions and growth. Cambridge, Mass.: The MIT Press.

Nolan, P. y Yeung, G., 2001a. Big Business with Chinese Characteristics: Two paths to growth of the firm in China under reform. Cambridge Journal of Economics, 25(4), pp. 443-65.

Nolan, P. y Yeung, G., 2001b. Large Firms and Catch-up in a Transitional Economy: The case of Shougang Group in China. Economics of Planning, 34(1-2), pp. 159-78. 
Nolan, P. y Zhang, J., 2002. The Challenge of Globalization for Large Chinese Firms. World Development, 30(12), pp. 2089-107.

North, D.C., 1979. A Framework for Analyzing the State in Economic History. Explorations in Economic History, 16(3), pp. 249-59.

North, D.C., 1993. The New Institutional Economics and Development. [pdf] Disponible en: $<$ http://ideas.repec.org/p/wpa/wuwpeh/9309002.html> [Consultado el 25 de junio de 2012].

North, D.C., 1995. Instituciones, cambio institucional y desempeño económico. México: Fondo de Cultura Económica (FCE).

North, D.C. y Thomas, R.P., 1973. The Rise of the Western World: A new economic bistory. Cambridge: Cambridge University Press.

Pamlin, D. y Baijin, L., 2007. Rethink China's Outward Investment Flows. [en línea] Switzerland: World Wildlife Fund. Disponible en: < http://wwf.panda.org/who_we_are/ wwf_offices/china/news/index.cfm?uGlobalSearch $=$ rethink $\% 20$ china $\% 27 \mathrm{~s} \% 20$ outward $>$ [Consultado el 23 de junio de 2012].

Peng, M. W., 2005. Perspectives: from China strategy to global strategy. Asia Pacific Journal of Management, 22(2) pp. 123-41.

Rui, H. y Yip, G.S., 2008. Foreign Acquisitions by Chinese Firms: A strategic intent perspective. Journal of World Business, 43(2) pp. 213-26.

Rutherford, M., 2001. Institutional Economics: Then and now. The Journal of Economic Perspectives, 15(3), pp. 173-94.

Sauvant, K.P., 2005. New Sources of FDI: The BRICs, outward FDI from Brazil, Russia, India and China. Journal of World Investment and Trade, 6(5), pp. 639-709.

Scott, B.R., 1985. National Strategies: Key to international competition. En: Scott, B.R. y Lodge, G.C., eds. 1985. U.S. Competitiveness in the World Economy. Boston: Harvard Business School Press.

Shan, W., 1989. Reforms of China's Foreign Trade System: Experiences and prospects. China Economic Review, 1(1), pp. 33-55.

Sheahan, J. B., 1976. Public Enterprise in Developing Countries. En: Sheperd, W., ed. Public Enterprise: economic analysis of theory and practice. Lexington, Mass.: HeathLexington Books.

Sinochem, 2012. Products and services-Finance. [en línea] Disponible en: $<$ http://english. sinochem.com/g749.aspx > [Consultado el 23 de junio de 2012].

Teagarden, M. y Cai, D., 2008. Learning from Dragons who are Learning from Us: Developmental lessons from China's global companies. Organizational Dynamics, 38(1), pp. 73-81.

Trade Lawyers Advisory Group, 2007. The Crisis in Intellectual Property Protection and China's Role in that Crisis. [en línea] Washington, D.C.: Trade Lawyers Advisory Group. Disponible en: <http://www.uscc.gov/researchpapers/2008/TLAG\%20Report $\% 20$ - 
$\% 20$ Crisis $\% 20$ in $\% 20 \mathrm{IP} \% 20$ Protection $\% 20$ and $\% 20$ China's\%20Role.pdf > [Consultado el 27 de mayo de 2012].

Walters, K.D., y Monsen, R.J., 1977. The Nationalized Firm: The politicians free lunch? Columbia Journal of World Business, 12(1), pp. 90-102.

Wang, M.Y., 2002. The Motivations behind China's Government-initiated Industrial Investments Overseas. Pacific Affairs, 75(2), pp. 187-206.

Williamson, O.E., 1989. Las instituciones económicas del capitalismo. México: FCE.

Wu, H.L. y Chen, C.H., 2001. An Assessment of Outward Foreign Direct Investment from China's Transitional Economy. Europe-Asia Studies, 53(8), pp. 1235-54.

Wu, D. y Zhao, F., 2007. Entry Modes for International Markets: Case study of Huawei, a Chinese technology enterprise. International Review of Business Research Papers, 3(1), pp. 183-96.

Zhang, H., 2008. Corporate Governance and Dividend Policy: A comparison of Chinese firms listed in Hong Kong and in the Mainland. China Economic Review, 19(3), pp. 437-59.

Zhou, Y., 2008. Synchronizing Export Orientation with Import Substitution: Creating competitive indigenous high-tech companies in China. World Development, 36(11), pp. 2353-70. 